\title{
The evidence for reducing inferior turbinates*
}

\author{
David Willatt
}

ENT Departments of Salford Royal NHS Foundation Trust, Central Manchester and Manchester Children's University Hospitals, Manchester, United Kingdom

SUMMARY

\begin{abstract}
Nasal obstruction is commonly due to enlargement of the inferior turbinate. This review discusses the pathophysiology of turbinate enlargement, the indications for, and methods and outcome of turbinate reduction. All techniques are successful but vary in their long-term efficacy, their propensity for complications and the degree to which they may adversely affect nasal function. Newer techniques under local anaesthetic and often endoscopic control offer outpatient treatment with satisfactory outcomes. However selecting a particular technique should take account of the individual patient's features, the surgeon's experience and judgement and informed patient choice.
\end{abstract}

Key words: inferior turbinate, turbinate reduction, nasal blockage, turbinate surgery

\section{INTRODUCTION}

Although the inferior turbinate may be operated on for reasons of neoplasia, epistaxis, and a source of graft material, by far the commonest reason is for chronic nasal obstruction. Chronic nasal obstruction is a common symptom of nasal disease and has many adverse sequelae including mouth breathing, dryness of the oropharynx, nasal speech, disordered sleep, restlessness, malaise, an adverse effect on quality of life and reduced lung volumes ${ }^{(1-3)}$. Inferior turbinate enlargement due to rhinitis is one of the main causes of chronic nasal obstruction ${ }^{(4)}$. The rhinitis may be allergic, infective, vasomotor, hormonal or secondary to medication ${ }^{(5,6)}$. Fortunately the turbinate enlargement is usually amenable to medical treatment of the underlying inflammatory process ${ }^{(7)}$. After establishing a diagnosis, medication can be started, an aggravating medication discontinued, a responsible allergen avoided or hyposensitisation commenced ${ }^{(6)}$. Maximum relief often requires corticosteroids ${ }^{(6)}$ and antihistamines ${ }^{(8)}$. However, long standing swelling may become irreversible. This may be due to dilated submucosal venous sinuses becoming varicose and unresponsive to sympathetic nervous system stimulation or medical treatment ${ }^{(9)}$ or because of fibrosis ${ }^{(10-12)}$. As such some patients are refractory to medical management or show only slight response and complain of persistent symptoms ${ }^{(13)}$. Alternatively some patients prefer surgical treatment ${ }^{(8)}$. In these instances inferior turbinate surgery may be offered ${ }^{(6,8,14,15)}$. Turbinate surgery is common and has been reported as the eighth most common procedure performed by otolaryngologists ${ }^{(16)}$.

\section{PATHOPHYSIOLOGY OF TURBINATE ENLARGEMENT}

The physics established with Poiseuilles law demonstrates that as little as a $10 \%$ increase in the cross sectional area of the nasal passage produces a $21 \%$ increase in flow. Studies using external nasal dilators have shown $70 \%$ of the effects on nasal resistance can be achieved with external dilators in the region of the nasal valve and anterior region of the inferior turbinate ${ }^{(17)}$. Decongestion of the nose increases the volume of the nasal cavity by $35 \%{ }^{(18)}$. Given the predominant effect of decongestants is vasoconstriction of the inferior turbinate, and given the anterior portion of the inferior turbinate is intimately associated with the region of the nasal valve area, inferior turbinate reduction should produce a significant change in nasal obstruction.

Whereas enlargement of the inferior turbinate is a common cause of nasal obstruction, enlargement of the middle turbinate is not. Inferior turbinate enlargement can be bilateral or unilateral. Bilateral is due to hypertrophy from allergic or nonallergic rhinitis. Unilateral enlargement occurs in association with a congenital or acquired anatomical deviation of the septum into the contralateral nasal passage. Such unilateral enlargement is thought to be secondary to the septal deviation, with the larger turbinate protecting the more patent nasal passage from excessive airflow, which may dry the nasal mucous membrane resulting in crusting. A second theory is primary growth of one inferior turbinate, genetic or as a result of trauma in early life, pressing on the growing septum in early life causing it to bend. The unilateral enlargement occurs as a result of increase in the size of the mucosa and conchal bone (19). 
The central strategy of turbinate surgical procedures is to reduce the volume of the inferior turbinate particularly in its anterior portion, a component of the internal nasal valve, the most resistive segment of the upper airway. The nasal airflow is to be improved without affecting nasal physiology. The inferior turbinate plays a major role in filtering, warming and humidifying inspired air, directing airflow particularly up into the olfactory area, and protecting the host with mucosal IgA and mucociliary clearance. The mechanism of perception of airflow is not fully understood, in particular the presence of airflow receptors in the mucosa has been a subject of controversy. Although nasal obstruction is commonly associated with an increased nasal airway resistance, the objective measurement of nasal airway resistance does not always correlate with subjective degree of nasal obstruction. Damaged, resected or by-passed trigeminal nerve endings can create the sensation of nasal obstruction despite no objective increase of nasal airway resistance. On the contrary, stimulation of the menthol receptors can improve the subjective sensation of nasal airflow without a decrease in airway resistance ${ }^{(20)}$. Histologically, tactile, pressure and thermoreceptors have been identified in the nasal mucosa. The cooler the nasal lining the clearer the nose feels supporting a hypothesis that the sensation of nasal airflow is derived from a cooling of the nasal lining on inspiration, which is probably detected by cold thermoreceptors ${ }^{(21)}$. Jones and colleagues demonstrated that anaesthesia of the nasal vestibule had a more pronounced effect on airflow sensation than anaesthesia of the nasal mucosa ${ }^{(22,23)}$, but Clarke demonstrated the nasal lining is sensitive to an air jet ${ }^{(24)}$ with Wrobel and colleagues finding the inferior meatus to be most sensitive ${ }^{(25)}$. From a clinical standpoint, understanding the distribution of the sensitivity of the nasal mucosa to airflow is important in preserving specific regions of nasal mucosa during surgical intervention to help to maintain the perception of airflow. The inferior turbinate takes part in the transient asymmetric congestion, as in the nasal cycle, and symmetric congestion in response to various stimuli to warm, humidify and filter inspired air. Such congestion is an important physiological function of the nose and needs to be preserved in performing surgery.

Therefore the ideal turbinate reduction procedure should reduce inferior turbinate volume, preserve the overall turbinate shape responsible for shaping an aerodynamic air stream, preserve physiological functions while minimising iatrogenic complications. Any technique destroying the turbinate mucosa is more likely to lead to a loss of turbinate function, crusting and adhesions ${ }^{(26-28)}$.

Understanding the histology of the hypertrophic inferior turbinate is imperative for the development and management of inferior turbinate reduction surgery. A study by Berger and colleagues compared pathologically hypertrophied inferior turbinates with normal turbinates ${ }^{(14)}$. The normal turbinate consists of an outer layer of respiratory mucosa, a submucosal layer, an inner periosteal layer and the turbinate bone. The submucosal layer is largely venous sinusoids, capable of engorgement with blood, causing swelling of the nasal mucosa. The principal control of the distension of the venous sinusoids is by the sympathetic nervous system and alters the response to various physiological and pathological factors. The pathologically hypertrophied inferior turbinate is quantitatively significantly wider, with the medial mucosal layer doubling in width and making the greatest contribution to the hypertrophy of the inferior turbinate, the bone changing very little in size. Qualitatively the hypertrophied inferior turbinate shows dilated, engorged thin walled venous sinusoids, marked subepithelial inflammatory cell infiltrate beneath the basement membrane and fibrosis of the lamina propria, suggesting a progressive and irreversible course and representing the end point of inflammation. At this point supportive treatment usually fails and the grounds for surgical reduction are laid down. On the basis of these findings Berger and colleagues suggest targets for inferior turbinate reduction surgery are the medial and inferior mucosal layers. The medial layer shows the greatest thickening and obstructs the airway. The inferior layer contributes less to the increase in width but is rich in sinusoids, poor in glandular elements and lacks major arteries. Therefore surgical reduction of the inferior layer will help congestion, will not increase the risk of nasal dryness nor increase the risk of perioperative haemorrhage. They feel the lateral mucosal layer should be spared as it does not encroach on the airway and has an important role in humidifying the inspired air and maintaining mucociliary clearance ${ }^{(14)}$.

Histological study of the inferior turbinates in patients with a deviated septum and compensatory enlargement of the contralateral inferior turbinate shows not only some increase in the thickness of the mucosa but also a significant doubling in the thickness of the bone ${ }^{(29)}$. Increase in the bony layer accounts for $75 \%$ of the entire growth of the inferior turbinate. CT scanning suggests this increase in the bony layer takes place in the anterior and middle thirds of the turbinate compared with the contralateral side ${ }^{(30)}$. As Farmer and Eccles have pointed out, an appreciation of the relative enlargement of the bony and soft tissue constituents of the inferior turbinate is important when deciding on which type of surgical intervention is most appropriate ${ }^{(15)}$.

\section{INFERIOR TURBINATE SURGERY}

When do we operate on hypertrophied inferior turbinates? Only after a thorough trial of medical treatment, which is to include topical nasal steroids, given over a protracted length of time has failed ${ }^{(31)}$. In a consensus statement in 2003, a trial of medical management is described as mandatory before surgery is undertaken ${ }^{(32)}$. However, the duration and exact nature of medical treatment is not well defined. Studies are required to establish what is sufficient medical treatment and how long it should be administered for before surgery is considered.

Various surgical treatments for enlarged inferior turbinates have been tried over the centuries. The selection of an opera- 
tive procedure to treat the inferior turbinate in chronic rhinitis remains controversial.

\section{Submucosal Diathermy}

Submucosal diathermy has been one of the most popular procedures. Submucosal diathermy is peformed under general anaesthesia. The nose is first decongested. Diathermy is performed using an insulated needle electrode. The needle tip is pressed against the anterior end of the inferior turbinate and activated for a short period giving a devascularised zone, to reduce bleeding. The needle is then introduced submucosally through this zone to the posterior end of the turbinate, care being taken to stay close to the bone. The diathermy is then turned on whilst the needle is slowly withdrawn over a period of 5 seconds. Three such passes are performed for each inferior turbinate at a coagulation current of $70 \mathrm{~W}$. If the diathermy current is sufficient, the mucosa of the turbinate blanches and shrinks. There is rarely any need for nasal packing allowing the procedure to be performed as a day case. Submucosal diathermy produces its effects by applying an alternating current in a megahertz frequency range to the tissue. The high rate of change in current in a volume conductor, in this case the inferior turbinate, leads to heating of the tissue. The heating is greatest where current density is highest, which is at the tip of the diathermy needle and leads to a submucosal burn. The submucosal burning leads to subsequent scarring, fibrosis and obliteration of the venous sinusoids. Engorgement is thus largely abolished causing a decrease in the volume of the turbinate mucosa and relief of airway obstruction. Initially, however, the thermal damage leads to an inflammatory response with a period of worsening of the obstruction before improvement occurs, with a dramatic fall in resistance at 2 months ${ }^{(33)}$. Topical vasoconstrictor drugs contain alpha adrenoreceptor agonists which cause vasoconstriction in the nasal submucosa manifested as collapse of the venous sinusoids. This collapse leads to nasal mucosal shrinkage and corresponding increase in nasal patency. The classical concept is that submucosal diathermy will only be effective if the inferior turbinates are capable of decongestion. Indeed a study by Jones and colleagues demonstrated a good correlation between both the objective and subjective reduction in nasal resistance achieved pre-operatively with vasoconstrictors, and the reduction of nasal resistance achieved by submucosal diathermy ${ }^{(10)}$. It has been suggested that submucosal diathermy would not be effective in a group of patients developing hypertrophic rhinitis where the nasal submucosa is infiltrated with fibrous tissue rendering it incapable of decongestion ${ }^{(10-12)}$. However, it has been hypothesised that in chronic perennial rhinitis with nasal obstruction the venous sinusoids become atonic losing the ability to vasoconstrict with either endogenous or exogenous adrenergic stimulation ${ }^{(9)}$. As a result submucosal diathermy would be effective even though preoperative assessment of nasal vasoconstriction would not necessarily be predictive. Further, some surgeons specifically utilise submucosal diathermy when the mucosal swelling is refractory to adrenergic agonists.

Whereas Sherman found that patients with nasal allergy were more often dissatisfied after nasal surgery for obstruction than were patients with non allergic rhinitis ${ }^{(34)}$, Jones found there was no tendency for patients with allergic rhinitis to suffer a poor result from submucosal diathermy surgery either objectively or subjectively ${ }^{(33)}$.

However, although submucosal diathermy provides good relief from nasal obstruction in the short term, Jones and Lancer showed that in patients who do not have concurrent medical treatment in most the inferior turbinates rehypertrophy within 15 months and subjective obstruction returns ${ }^{(31)}$. Similarly, a study by Warwick-Brown and Marks of over 300 patients treated with submucosal diathermy or linear cautery demonstrated subjective improvement in $82 \%$ of patients at one month, but falling to $54 \%$ at one year ${ }^{(35)}$.

\section{Radical Turbinectomy}

If submucosal diathermy confers only short-term efficacy, perhaps complete removal of the inferior turbinate at the outset should be considered. Radical turbinectomy halves the nasal resistance to airflow initially ${ }^{(36)}$. At 2 years only $20 \%$ of patients complain of recurrent obstruction ${ }^{(37)}$. Ophir reported only $20 \%$ of his patients undergoing radical turbinectomy failed to notice an improvement in their nasal breathing, which corresponds with Wight et al.'s results ${ }^{(38)}$. However the procedure is attended by a significant risk of severe reactionary haemorrhage in up to 10 per cent of patients ${ }^{(39)}$, and is associated with more postoperative pain than other techniques ${ }^{(8)}$. Traditionally, concern has been raised that total removal of hypertrophied turbinates may result in rhinitis sicca and /or ozaena ${ }^{(40)}$. These processes have been attributed to excessive drying from loss of turbinate mucosa, destruction of cilia secondary to scarring, atrophy and endstage infection ${ }^{(41)}$. The term ozaena has been applied to a foul smelling nasal discharge, the aetiology of which has not been established. Several organisms have been incriminated, including Klebsiella ozenae, a form of Clostridium diphtheriae and the Perez-Hofer bacillus, but these are more likely secondary invaders of necrotising tissue ${ }^{(42)}$. The results of studies on the postoperative risk of atrophic rhinitis and /or ozaena vary. In 1983, Martinez reported on 29 patients followed up from 2 to 60 months postoperatively by clinical examination and by formal questionnaire ${ }^{(43)}$. Twenty-five patients described marked improvement of their nasal breathing, 3 had mild improvement and 1 had no improvement. Only one patient 1-year postoperatively described excessive dryness, 2 described mild dryness, 3 described excessive secretions and none complained of foul smell or pain postoperatively. All patients had patent airways by clinical examination. Carrie et al. reported on the long-term benefit of radical trimming in 14 patients at least 7 years post operatively ${ }^{(44)}$. They found that although nasal obstruction may have recurred to some extent there was still significantly lower nasal resistance than the pre-operative 
state. Thirty-five per cent complained of nasal crusting, particularly in the mornings, which they had not complained of preoperatively. Fifty per cent were noted to have crusting at the back of the nose at the time prior to rhinomanometric evaluation. However no patients had atrophic rhinitis or ozaena.

Conversely, Moore and colleagues followed up for between 2 and 7 years 18 patients who had undergone total inferior turbinectomy. Only 11\% were symptom free, $66 \%$ had chronic nasal crusting causing them to detect a nasal odour consistent with a diagnosis of atrophic rhinitis, and $22 \%$ had chronic nasal crusting and a nasal odour detectable by others but not by the patient because of olfactory dysfunction consistent with ozaena (45). Moore's findings support the traditional point of view that radical turbinectomy may result in rhinitis sicca and/or ozaena. Some authors have recognised a symptom of nasal congestion after turbinectomy in the absence of anatomic obstruction on examination or CT. This has been termed the 'empty nose syndrome', a term first used in the Mayo Clinic by Kern and Stenquist in 1996. The 'empty nose syndrome' has been attributed to the patient's inability to recognise the normal baseline nasal sensation of breathing. The cause is unknown but a number of causes have been postulated ${ }^{(46)}$. It is already known from electron microscopy studies that patients with atrophic rhinitis have atrophy of the olfactory epithelial receptors leading to anosmia. It is possible they have atrophy of the nerve fibres in the nasal lining subserving the sensation of nasal airflow. Alternatively, resecting the sensate turbinate deprives the patient of sensation of airflow. Furthermore, the obliteration of all nasal resistance may not result in attainment of satisfactory nasal respiration. Nasal breathing is more satisfying than mouth breathing. The nose provides half the resistance of the entire respiratory tract. This resistance may need to balance the pulmonary resistance during inspiration. An extreme lack of nasal resistance may paradoxically be perceived as nasal obstruction. In one study the effects of excess removal of turbinate tissue (namely the empty nose syndrome, crusting, bleeding, recurrent infections, nasal odour, pain and /or clinical depression) occurred at a mean time of longer than 8 years following turbinate surgery ${ }^{(32)}$.

For the above reasons total inferior turbinate resection has been falling out of favour. Most otorhinolaryngologists now believe that turbinate resection should be performed as conservatively as possible, but are willing to do what is necessary to achieve the desired result ${ }^{(32)}$.

\section{Partial Turbinectomy}

Anterior turbinectomy removes less tissue, offers the attraction of reducing airway resistance in the critical region of the nasal valve and avoids the large branches of the sphenopalatine artery, which enter the turbinate posteriorly. However, Wight, Jones and Clegg showed anterior trimming of the inferior turbinate, whilst being an objective success in decreasing nasal resistance, frequently failed to produce a significant fall in subjective obstruction in the first 2 months ${ }^{(47)}$. Even in patients who had relief of symptoms, the turbinate soon rehypertrophied ${ }^{(37)}$. This contrasts with a study by Fanous, who reported a good to excellent improvement after anterior turbinectomy in patients followed up for 6 months to 4 years ${ }^{(48)}$.

Turbinoplasty is a form of partial turbinate resection. In 1982 Mabry described his method of turbinoplasty because of disappointment with submucosal diathermy ${ }^{(49)}$. The inferior turbinate is infractured, a 15 inch blade is used to make an incision down to conchal bone and the soft tissue is elevated off the medial surface. The conchal bone and a wedge of stroma attached to the lateral and inferior aspect is resected with scissors. The remaining medial flap is rolled up on itself from medial to lateral to form a neoturbinate with two apposing inverted raw surfaces and an external mucosal surface. In his series all patients additionally had septoplasty. At 1-year follow-up, all had a marked improvement with no nasal crusting, dryness or bleeding. Even 5 or more years after the inferior turbinoplasty, Mabry found no evidence of atrophic rhinitis ${ }^{(50)}$. He found the major difference between total turbinectomy and inferior turbinoplasty was the flap of tissue left behind in the latter procedure to form a neoturbinate. As his histological analysis showed insignificant mucus secreting glands in the flap, he postulated the functional difference in the two procedures is explained by the presence of the inferior neoturbinate acting as a baffle to preserve a more normal airflow ${ }^{(50)}$. He indicated failure to deal with the original cause of turbinate hypertrophy could and probably would result in recurrent turbinate hypertrophy, reinforcing the need for appropriate and continuing medical management of all patients with such nasal problems.

Degloving is a partial turbinectomy technique requiring general anaesthesia and packing ${ }^{(8)}$. It offers the advantage of removing hypertrophied turbinate tissue along the whole length of the inferior turbinate, whilst leaving turbinate bone intact. Subjective improvement in nasal patency was maintained at 2-year follow-up in $89.5 \%$ of patients. Rhinorrhoea was reduced and sense of smell increased. There was no significant change in mucociliary clearance as judged by saccharin clearance and no haemorrhagic complications in the group studied.

Garth and colleagues showed that whereas in partial inferior turbinate resection synechiae occur in up to $10 \%$ of patients, the risk of bleeding is decreased from $5.8 \%$ to $0.9 \%$ when compared to total turbinectomy ${ }^{(51)}$.

However, trimming remains a controversial procedure particularly in relation to the possible effects of the operation on nasal physiology.

\section{Outfracturing}

What conservative methods are available? Crushing and lateral outfracture is the simplest surgical method of treating enlarged inferior turbinates. This is performed by medially infracturing the turbinate, crushing it with a flat bladed instrument, then forcing it laterally and holding it in position temporarily with nasal packing. Goode indicates that not only does the turbinate 
tend to eventually return to its original position, but the procedure also fails to deal with the primary cause of its enlargement ${ }^{(52)}$.

\section{Cryotherapy}

Cryotherapy of the inferior turbinates was first introduced by Ozenberger in $1970{ }^{(53)}$. It is usually performed under local anaesthesia and apart from a transient headache, is not usually associated with significant post-operative adverse sequelae. In our series we used topical cocaine but now use a topical lignocaine and adrenaline spray for local anaesthesia, after which the cryoprobe can be applied to each inferior turbinate at between 2 - 4 points, each application lasting 90-120 seconds ${ }^{(54)}$. Cryotherapy exerts its effects by the intracellular formation of ice crystals and subsequent cell membrane destruction. The critical temperature is -12 degrees Celsius. Thrombosis of small vessels and subsequent ischaemia increase the tissue destruction and the effectiveness of cryotherapy. Effectiveness is increased by longer treatment duration, larger areas of therapy and repeated treatment sessions. There is a reduction of the post operative ability of the nasal mucosa to decongest as compared with submucosal diathermy implying the procedure is more effective than submucosal diathermy in obliterating venous erectile tissue. In our group of patients, 54\% obtained relief of their symptoms at between 9 and 33 months (median 22 months) follow up ${ }^{(54)}$. Many authors have drawn attention to the importance of adequate patient selection in order to maximise the effectiveness of cryotherapy ${ }^{(55,56)}$. Cryotherapy will not alleviate nasal obstruction secondary to turbinate bone hypertrophy or major septal deviation. While we found greater success in patients with allergy than without and slightly worse when a minor septal deviation was present, these differences were not significant. Thus, unlike some authors ${ }^{(56,57)}$, we continue to offer cryotherapy as one of our methods of turbinate reduction when there is an allergic component to the symptoms or a minor septal deviation. In many cases cryotherapy avoids more radical turbinate surgery, indeed cryotherapy can be repeated without ill effect.

\section{Laser reduction}

Recent advances in turbinate surgery have allowed other day case procedures, often under local anaesthesia, with minimal morbidity and no nasal packing.

The beam of coherent light of a laser may be accurately delivered producing minimal damage beyond the area requiring treatment. Carbon dioxide, diode, neodymium-yttrium aluminium garnet (Nd:YAG), potassium-titanyl-phosphate (KTP), argon-ion, and holmium-yttrium aluminium garnet (Ho:YAG) laser have been used to treat hypertrophied inferior turbinates ${ }^{(58)}$. Apart from the carbon dioxide laser, all lasers can be delivered down a flexible quartz fibre in a contact or non-contact mode. The carbon dioxide laser produces energy in the infrared wavelength and is highly absorbed by water molecules within the tissues, producing heat and surface ablation. Cook,
McCombe and colleagues found it to be superior to submucosal diathermy with less post-operative morbidity and maintenance of subjective patency at 1-year follow-up ${ }^{(59)}$. They used an arthroscopic fitment, facilitating laser treatment from the posterior end of the turbinate along the turbinate axis. In Elwany and Harrison's study, using the laser sighted through a microscope the only complication encountered with laser treatment was synechiae formation. For that reason they advised avoiding vaporisation of the medial surface of the inferior turbinate beyond its anterior third ${ }^{(60)}$. We studied a series of children undergoing carbon dioxide laser vaporisation reduction of the inferior turbinates, again with the laser sighted through an operating microscope. We found $90 \%$ of children had a clear nose at $18-24$ month post operatively ${ }^{(61)}$. Although Sapci and colleagues ${ }^{(28)}$ found the carbon dioxide laser disturbed mucociliary function, we found postoperative mucociliary clearance, as assessed by saccharin clearance, to be normal ${ }^{(61)}$. In our $10 \%$ of patients who had recurrent nasal obstruction, the turbinates were trimmed and histologically examined to show healthy ciliated reepithelialisation of the mucosal surface (61). KTP laser light and argon laser light are absorbed by endogenous chromophores such as haemoglobin and melanin. Coagulation depth is up to $2 \mathrm{~mm}$. Orabi and colleagues ${ }^{(62)}$ reported on 39 patients with either allergic or non-allergic rhinitis who had KTP laser treatment to their inferior turbinates under local anaesthesia. In all there was an initial improvement in blockage, hyposmia, rhinorrhoea and sneezing. At 6 months only 28\% required medication to aid symptom control. Wang and colleagues, again using a KTP laser, detail $87 \%$ of patients reporting improvement in nasal obstruction with a mean follow-up of just over two years ${ }^{(63)}$. Ferri and colleagues followed up 157 patients treated with Argon plasma surgery for turbinate hypertrophy. After 24 months $87 \%$ of patients reported improved nasal airflow as compared to preoperatively ${ }^{(64)}$. To reduce mucosal damage Supiyaphun and colleagues used an 18-gauge needle to introduce an optical fibre into the inferior turbinate. Retrograde photocoagulation was performed while the fibre was slowly withdrawn from the nose. Sneezing, rhinorrhoea, itching and obstruction were reduced post operatively as assessed by the patient and clinician ${ }^{(65)}$. A review of the literature concludes laser treatment of the hyperplastic inferior turbinate can be considered a useful, cost effective, time saving procedure which can frequently be performed in out patients under local anaesthesia, with short operation time, good results and minor side effects. However, the review indicated that whereas, in published studies this procedure achieves comparable or better results than more conventional techniques, it appears to be less effective in the long-term ${ }^{(58)}$.

\section{Radiofrequency reduction}

Described by Li and colleagues in $1998^{(66)}$, radiofrequency reduction of the inferior turbinates has emerged as a new and possibly cost effective day case, local anaesthetic treatment. 
Through targeted and controlled dosing it creates a submucosal lesion that allows for the presence of mucociliary function whilst achieving tissue volume reduction of the submucosa. The submucosal lesion results from friction between ions, which rapidly change direction because of an alternating current produced by the electrode. Heat is generated in the surrounding tissue rather than at the tip of the electrode. A temperature of less than 85 degrees centigrade is generated as opposed to the 800 degree temperature brought about by electrocautery or laser. The lesion is therefore controlled and the overlying mucosa, its mucociliary clearance and the underlying turbinate bone are preserved. Post-operative wound contracture and fibrosis induces tissue volume reduction. The probe, which usually has a distal active portion and a proximal insulated portion, is inserted into the turbinate. Many studies report subjective improvement in nasal obstruction by the patient and clinician after radiofrequency turbinate reduction. A randomised, blind, placebo controlled trial showed radiofrequency treatment to be significantly better than placebo at 6 months ${ }^{(67)}$. Harsten ${ }^{(68)}$ reported on 158 patients treated with radiofrequency (Surgitron IEC, Ellman International, Inc., New York, NY, USA) set at bipolar coagulation mode, followed up by questionnaire between 3-30 months post-operatively. The study reports a large beneficial effect with complete relief or definite subjective improvement in nasal obstruction in $85 \%$ of patients. This was irrespective of a short-term or a long-term follow-up and was applicable to patients who additionally had septal deviations.

Kizilkaya and colleagues ${ }^{(69)}$ found a significant improvement in visual analogue scale scores and acoustic rhinomanometry 12 weeks and 6 months following radiofrequency treatment to hypertrophic inferior turbinates. Cavaliere, Mottola and Iemma ${ }^{(70)}$ evaluated the efficacy and morbidity of monopolar Somnoplasty (Somnus Medical Technologies, Inc, Sunnyvale, CA, USA) and bipolar "Coblator II ENT" (Arthrocare Corp, Sunnyvale, CA, USA). They found both methods of radiofrequency volumetric reduction brought about a long-term improvement in symptoms and maintanace of nasal function lat 20 months post surgery. Porter and colleagues reported sustained benefit of radiofrequency treatment of hypertrophied inferior turbinates at 2 years postoperatively ${ }^{(71)}$. Barbieri and colleagues reported the technique was valid, effective and safe in 1689 non-allergic patients treated with radiofrequency ${ }^{(72)}$. In Rhee and colleagues' study, not only was there a significant reduction in nasal resistance via rhinomanometry, but even as early as 1 week postoperatively, no changes in saccharin transit time or ciliary beat frequency were seen, implying that radiofrequency techniques did not disturb mucociliary function ${ }^{(73)}$. In patients with persisting nasal obstruction, a second surgical attempt with radiofrequency turbinectomy can be successful, in contrast to previous reports on turbinectomy performed by laser ${ }^{(68)}$.

\section{Powered turbinate reduction}

With the advent of powered instruments, turbinoplasty has enjoyed renewed interest. Joniau and colleagues describe an extraturbinal turbinoplasty ${ }^{(5)}$. The use of an endoscope provides unequalled visualisation of the surgical field and the ability to remove tissue precisely. Under general anaesthesia the anterior end and inferior posterior border is infiltrated with $2 \%$ lignocaine and 1 in 80,000 adrenaline. If necessary, the turbinate is fractured medially, allowing space for both a zero degree endoscope and powered microdebrider to access the lateral surface of the inferior turbinate. A microdebrider with a straight blade is used in an oscillate mode to remove the soft tissue from the lateral aspect of the vertical portion of the inferior turbinate. The majority of the turbinate bone is then removed from this bony lamella. Residual bone fragments are dissected free with a malleable probe and paediatric backbiter. After all the lateral mucosa and bone has been removed, the remaining mucosa is rolled on itself using a Freer's elevator.

A rectangular sheet of Surgicel (oxidised cellulose; Ethicon, Inc., Somerville, NJ, USA) may be used to cover the turbinate and prevent unrolling. No postoperative packing is necessary. With this technique they showed long term subjective and objective relief of nasal obstruction at 5-year follow-up. Wu and colleagues showed 70 patients had significant improvement in nasal obstruction evaluated by visual analogue scale and acoustic rhinomanometry at 12 months post operatively, though allergic patients had less favourable results ${ }^{(74)}$.

With the advent of a newly designed small $2 \mathrm{~mm}$ microdebrider blade incorporated with an elevator, intraturbinal powered submucosal turbinate reduction is now available. Under local anaesthesia, the turbinate can be injected with lignocaine and adrenaline. A vertical incision is made with a number 11-inch blade in the anterior aspect of the inferior turbinate and a submucosal pocket is created with a sharp dissector on the medial surface of the bony turbinate. A straight $2 \mathrm{~mm}$ microdebrider with an elevator is inserted with further elevation with the dissector. Debridement of the submucosal tissue from the inferior turbinate is performed with the blade positioned laterally in the submucosal plane at speeds of up to $3000 \mathrm{rpm}$. More aggressive resection can be performed by positioning the blade facing medially. No postoperative packing is necessary. Yanez ${ }^{(75)}$ noted an initial 1-month period of postoperative congestion in most patients. Huang found a significant decrease in nasal resistance and increase in quality of life at 1-year post operatively ${ }^{(2)}$. Rhinorrhoea, sneezing and post-nasal discharge improved significantly postoperatively in allergic patients which he felt was as a result of removal of the large amount of inflammatory cells in the medial submucosal tissue. In addition, submucosal turbinectomy severs branches of the postnasal nerve arising from the sphenopalatine foramen, which have a role in causing sneezing and hypersecretion ${ }^{(76)}$.

A 10-year follow-up by Yanez and Mora reported on 350 nonallergic patients treated by this submucous stroma debriding technique ${ }^{(77)}$. The study showed $91.3 \%$ of patients were com- 
pletely symptom free, 5.2\% had partial relief and only $3.25 \%$ had recurrence of nasal obstruction. Endoscopy, anterior rhinomanometry, and mucociliary transit time revealed long term improvements. Few complications were observed.

Functional inferior turbinosurgery (FITS) is powered intraturbinal submucous resection plus division of the posterior nasal nerve at the sphenopalatine foramen with placement of bony fragments at the foramen to prevent reinnervation ${ }^{(78)}$. Division of the post nasal nerve interrupts the parasympathetic and sympathetic fibres similar to vidian neurectomy and interrupts the somatic afferent fibres from the nasal mucosa, which can be expected to reduce the hypersensitivity and axon reflexes of the nasal mucosa, inhibiting neurogenic inflammation ${ }^{(79)}$. Ikeda and colleagues studied 56 patients who had either allergic or non-allergic rhinitis undergoing FITS ${ }^{(78)}$. They found $96 \%$ of patients had improvement in symptoms of nasal obstruction, sneezing, rhinorrhoea and quality of life at 1-year follow-up. All patients who underwent rhinomanometry showed postoperative improvement. Nasal provocation testing resulted in an apparently significant suppression of the antigeninduced allergic symptoms in all patients tested, indicating that allergic responses are inhibited by this operative procedure.

\section{Comparison studies}

Most studies analysing the various methods of inferior turbinate reduction are prospective in nature with short-term follow up periods. Few studies use a control and even fewer are randomised comparison studies.

Wengraf, Gleeson and Siodlak found submucosal diathermy to be as effective as cryotherapy ${ }^{(80)}$. Elwany and Harrison ${ }^{(60)}$ randomised 80 patients with chronic non-allergic rhinitis and hypertrophied inferior turbinates to four surgical treatments with a follow-up to one year. Partial inferior turbinectomy and laser turbinectomy improved nasal breathing in $77 \%$ of patients. The results of turbinoplasty and cryoturbinectomy were less favourable. The post-operative improvement in smell acuity correlated positively with the increased patency of the nasal airway. A comparison study by Rakover and Rosen ${ }^{(81)}$, with a follow-up of 2 to 5 years showed partial inferior turbinectomy maintained an improvement in nasal breathing in $77 \%$ of patients, whereas the effectiveness of cryosurgery fell from $62 \%$ at one year to $35 \%$ later.

Passali and colleagues ${ }^{(27)}$ conducted a large randomised clinical trial comparing total turbinectomy, carbon dioxide laser reduction, electrocautery, cryotherapy, submucosal resection and submucosal resection with lateral displacement. Submucosal resection with lateral displacement provided the best long-term results. Sapci and others ${ }^{(28)}$ found the efficacy of radiofrequency, carbon dioxide laser ablation and partial turbinectomy in relieving nasal obstruction to be the same at 12 weeks, though mucociliary transport time was significantly prolonged after laser treatment, and is one of the few studies to have had a control arm. In Joniau and colleagues study ${ }^{(5)}$ powered extraturbinal submucosal resection was superior to submucosal diathermy at 5-year follow-up. A significant difference was noted for postoperative crusting, endoscopic scoring of turbinate size, and acoustic rhinometry measurements of nasal cavity volume and mean area at the level of the nasal valve. In addition, the results of powered turbinoplasty were still apparent on long-term follow-up, whereas submucosal diathermy was associated with a recurrence of turbinate hypertrophy. In Lee and Lee's study ${ }^{(13)}$ powered extraturbinal turbinoplasty provided $80 \%$ of patients with symptomatic relief at 12 months, whereas radiofrequency coblation turbinate reduction gave a significantly lower relief of symptoms in $60 \%$ of patients at 12 months. Powered extra turbinal turbinoplasty resulted in a significantly larger cross-sectional area at the level of the head of the inferior turbinate and overall volume of the nasal cavity at 12 months postoperatively as compared to radiofrequency coblation reduction. In their opinion, when the anterior head and the inferior turbinate itself is severely hypertrophied, radiofrequency coblation was limited in its ability to reduce volume sufficiently. In contrast, the microdebrider effectively and precisely reduced the external surface of the hypertrophied inferomedial mucosa and the anterior head of the inferior turbinate. In their opinion, these were the reasons that cross-sectional area of the second notch and volume of nasal cavity, and the satisfaction of the patients in the microdebrider group were greater at 12 months postoperatively. In Kizilkaya and colleagues' study ${ }^{(69)}$ no difference was found between intraturbinal microdebrider and radiofrequency tissue volume reduction in inferior turbinate hypertrophy on assessing subjective patency, acoustic rhinomanometry, saccharin transport time, and ciliary beat frequency at 12 weeks and 6 months postoperatively.

\section{TURBINATE ENLARGEMENT CONTRALATERAL TO SEPTAL DEVIATION}

In most patients with a deviated septum, surgical correction enlarges the narrowed airway on the convex side of the deviation. However, this narrows the contralateral nasal passage. As there is pre-existing compensatory enlargement of the inferior turbinate on that contralateral side, some patients complain of nasal obstruction in the previously clear nostril. This is frequently predicted preoperatively and the enlarged inferior turbinate is surgically reduced at the time of septoplasty. Nasal resistance studies ${ }^{\left({ }^{(2)}\right.}$ with simulated septal deviation show that anterior obstruction increases the resistance to airflow more than posterior obstruction, and that total inferior turbinoplasty or total turbinectomy should not be necessary in connection with septoplasty. However, the evidence for any form of surgery to reduce the compensatorily enlarged turbinate alongside septoplasty is not well established. In a non-randomised trial of inferior turbinoplasty and septoplasty versus septoplasty alone Hilberg et al. ${ }^{(83)}$ found that two-thirds of patients who underwent contralateral turbinoplasty plus septoplasty reported satisfaction with the patency of the nasal cavity that had hadturbinate hypertrophy whereas none who underwent septo- 
plasty alone reported satisfaction with the patency of this nasal cavity. Whereas Illum ${ }^{(84)}$ reported that 5 years after surgery the effect of turbinate reduction was less apparent with satisfaction of patients who underwent septoplasty and turbinate reduction being similar to that of patients who underwent septoplasty alone. Inferior turbinate surgery alongside septoplasty is associated with increased morbidity primarily from haemorrhage and synechiae. The incidence of post-operative haemorrhage increases from $<2 \%$ to $6 \%$, and synechiae from $5 \%$ to $17 \%$ when turbinectomy is performed in addition to septoplasty ${ }^{(85)}$. Nunez and Bradley ${ }^{(86)}$ perfomed a prospective randomised study in 29 patients of septoplaty alone versus septoplasty and contralateral turbinectomy to establish whether this potential trebling in morbidity was justified. At 8 weeks postoperatively there was no difference in objective and subjective measures of nasal obstruction between the two groups. They concluded that surgical reduction of contralateral compensatory turbinate hypertrophy in patients with unilateral septal deviations is not associated with any additional benefit. This may be explained by the work of Kim et al. ${ }^{(87)}$ who studied the effects of septoplasty alone on the thicknesses and cross-sectional areas of mucosa and conchal bone with computed tomography before the operations and at least one year after surgery. They found that the thickening of the mucosa of the inferior turbinate on the concave aspect and the thinning on the convex aspect, especially in the medial mucosa, reverses after septoplasty. However, they did not identify how long this effect took to occur. No change in the thickness or dimensions of the conchal bone was noted on either side of the deviated septum in their study, but indicate further follow up was required to see if there are bony changes in the longer term. Stewart et al. ${ }^{(88)}$ found similar results to Nunez's study. In Stewart's study, 59 subjects were treated with septoplasty with or without turbinate reduction in a prospective, non-randomised multiinstitutional setting. Both the septoplasty with turbinate reduction group and the septoplasty alone group demonstrated clinically significant improvements from their respective baseline scores in a disease specific outcome instrument for nasal obstruction at 6 months. There was no significant difference between the two groups at 3 months and 6 months.

\section{CONCLUSION}

In conclusion, chronic nasal airway obstruction is commonly due to inferior turbinate hypertrophy. When medical management fails surgery may be effective treatment. However, the current indications for surgical treatment have been deemed empirical necessitating further research. The aims of surgery are to reduce the size of the turbinate to increase airflow, avoid complications and preserve nasal physiology. However, the mechanisms of perceiving unimpeded airflow are poorly understood. The inferior turbinate warms, humidifies and directs inspired air, exhibits reflexes and takes part in the nasal cycle. Surgery may adversely affect nasal function.

The large number of surgical techniques in use to reduce turbinate size indicates there is no single technique, which is effective in all patients. There is no'gold standard' nor is there any technique entirely free of side effects. Few areas of surgery are as controversial as the choice of technique to reduce the enlarged inferior turbinate. Generally, techniques which remove most turbinate tissue have the greatest and longest lasting effect, but are also accompanied by a higher likelihood of morbidity. Techniques include total or partial resection, diathermy, cryotherapy, laser therapy and radiofrequency ablation. Adverse side effects described include bleeding, synechiae, dryness, crusting, osteitis, foetor, atrophic rhinitis, the phenomenon known as 'empty nose syndrome' or inadequate volume reduction. Endoscopes are facilitating more precise surgery, and recent advances permit out patient procedures to be performed with minimal morbidity without nasal packing. The cause and degree of turbinate enlargement need to be assessed before choosing a particular technique. The underlying cause may necessitate postoperative medical management to prevent recurrent enlargement, and is preferable to radical surgery at the outset. Inappropriate selection of surgery or surgical modality as a therapeutic option appear to be major causes of patient dissatisfaction. The selection and recommendation of a particular surgical technique of inferior turbinate reduction, with its advantages and drawbacks, will be determined by the patient's clinical findings, the surgeon's experience and judgement and informed patient choice.

\section{REFERENCES}

1. Odetoyinbo O. Complications following total inferior turbinectomy: facts or myths? Clin Otolaryngol 1987; 12: 361-363.

2. Huang T, Cheng P. Changes in nasal resistance and quality of life after endoscopic microdebrider-assisted inferior turbinoplasty in patients with perennial allergic rhinitis. Arch Otolaryngol Head Neck Surg 2006; 132: 990-993.

3. Swift AC, Campbell IT, McKown TM. Oronasal obstruction, lung volumes, and arterial oxygenation. Lancet 1988; 1: 73-75.

4. Bridger GP, Proctor DF. Maximum nasal inspiratory flow and nasal resistance. Ann Otol Rhinol Laryngol 1970; 79: 481-488.

5. Joniau S, Wong I, Rajapaksa S, Carney SA, Wormald PJ. Long term comparison between submucosal cauterisation and powered reduction of the inferior turbinates. Laryngoscope 2006; 116: 16121616.

6. Mabry RL. Surgery of the inferior turbinates: How much and when? Otolaryngol Head Neck Surg 1984; 92: 571-576.

7. McCombe AW, Cook J, Jones AS. A comparison of laser cautery and submucosal diathermy for rhinitis. Clin Otolaryngol 1992; 17: 297-299.

8. Chevretton EB, Hopkins C, Black IM, Tierney P, Smeeton NC. Degloving of the inferior turbinates: pilot study to assess the effectiveness of a new technique. J Laryngol Otol 2003; 117: 866-870.

9. Richardson J. Turbinate treatment in vasomotor rhinitis. Laryngoscope 1985; 58: 834-847.

10. Jones AS, Wight RG, Kabil Y, Buckingham E. Predicting the outcome of submucosal diathermy to the inferior turbinates. Clin Otolaryngol 1989; 14: 41-44.

11. Cook PR. Sinusitis and allergy. Current Opinion in Otolaryngology \& Head and Neck Surgery 1997; 5: 35-39.

12. Eccles R. Nasal airflow in health and disease. Acta Otolaryngol 2000; 120: 580-595.

13. Lee JY, Lee JD. Comparative study on the long term effectiveness between coblation- and microdebrider- assisted partial turbinoplasty. Laryngoscope 2006; 116: 729-734. 
14. Berger G, Gass S, Ophir D. The histopathology of the hypertrophic inferior turbinate. Arch Otolaryngol Head Neck Surg 2006; 132: 588-594.

15. Farmer SEJ, Eccles R. Chronic inferior turbinate enlargement and the implications for surgical intervention. Rhinology 2006; 44: 234238.

16. Manoukian PD, Wyatt JR, Leopold DA, Bass EB. Recent trends in utilisation of procedures in Otolaryngology, head and neck surgery. Laryngoscope 1977; 107: 472-477.

17. Latte J, Taverner D. Opening the nasal valve with external dilators reduces congestive symptoms in normal subjects. Am J Rhinol 2005; 19: 215-219.

18. Grymer LF, Hilberg O, Pedersen OF, Rasmussen TR. Acoustic Rhinometry: values from adults with subjective normal patency. Rhinology 1991; 29: 35-47.

19. Egeli E, Demerci L, Yazycy B, Harputlouglu U. Evaluation of the inferior turbinate in patients with deviated nasal septum by using computed tomography. Laryngoscope 2004; 114: 113-117.

20. Eccles R, Morris S, Tolley NS. The effects of nasal anaesthesia upon nasal sensation of airflow. Acta Otolaryngol 1988; 106: 152155 .

21. Willatt DJ, Jones AS. The role of the temperature of the nasal lining in the sensation of nasal patency. Clin Otolaryngol 1996; 21: 519-523.

22. Jones AS, Crosher R, Wight RG. The effect of local anaesthesia of the nasal vestibule on nasal sensation of airflow and nasal resistance. Clin Otolaryngol 1987; 12: 461-464.

23. Jones AS, Wight RG, Crosher R, et al. Nasal sensation of airflow following blockage of nasal trigeminal afferents. Clin Otolaryngol 1989; 14: 285-289.

24. Clarke R, Jones AS, Charters P, et al. The role of mucosal receptors in the nasal sensation of airflow. Clin Otolaryngol 1992; 17: 387-392.

25. Wrobel BB, Leopold DA. Olfactory and sensory attributes of the nose. Otolaryngol Clin N Am 2005; 38: 1163-1170.

26. Hol MK, Huizing EH. Treatment of inferior turbinate pathology: a review and critical evaluation of the different techniques. Rhinology 2000; 38: 157-166.

27. Passali D, Passali FM, Damiani V, Passali GC, Bellusi L. Treatment of inferior turbinate hypertrophy: a randomised clinical trial. Ann Otol Rhinol Laryngol 2003; 112: 683-688.

28. Sapci T, Sahin B, Karavus A, Akbulut U. Comparison of the effects of radiofrequency tissue ablation, $\mathrm{CO} 2$ laser ablation, and partial turbinectomy applications on nasal mucociliary functions. Laryngoscope 2003; 113: 514-519.

29. Berger G, Hammel I, Berger R, Avraham S, Ophir D. Histopathology of the inferior turbinate with compensatory hypertrophy in patients with deviated nasal septum. Laryngoscope 2000; 110: 2100-2105.

30. Uzun L, Savranlar A, Beder LB et al. Enlargement of the bone component in different parts of compensatorily hypertrophied inferior turbinate. Am J Rhinol 2004; 18: 405-410.

31. Jones AS, Lancer JM. Does submucosal diathermy to the inferior turbinates reduce nasal resistance to airflow in the long-term? J Laryngol Otol 1987; 101: 448-450.

32. Rice DH, Kern EB, Marple BF, Mabry RL. Friedman WH. The turbinates in nasal and sinus surgery: A consensus statement. ENT-Ear, Nose and Throat Journal 2003; 82: 82-84.

33. Jones AS, Lancer JM, Moir AA, Stevens JC. The effect of submocosal diathermy to the inferior turbinates on nasal resistance to airflow in allergic and vasomotor rhinitis. Clin Otolaryngol 1985; 10 : 249-252.

34. Sherman AH. A study of nasal airway function in the post operative period of nasal surgery. Laryngoscope 1977; 87, 299-303.

35. Warwick-Brown NP, Marks NJ.Turbinate surgery: How effective is it? ORL 1987; 49: 314-320.

36. Wight RG, Jones AS. Radical trimming of the inferior turbinates and its effect on nasal resistance to airflow. J Laryngol Otol 1988; 102: 694-696
37. Wight RG, Jones AS, Beckingham E. Trimming of the inferior turbinates: a prospective long-term study. Clin Otolaryngol 1990; 15: 347-350.

38. Ophir D, Shapira A, Marshak G. Total inferior turbinectomy for nasal airway obstruction. Arch Otolaryngol Head Neck Surg 1985; 111: 93-95.

39. Goode RL. Surgery of the turbinates. J Otolaryngol 1978; 7:262268.

40. Gluckman JL. The Clinical Approach to Nasal Obstruction. In: Paparella MM, Schumrick DA. Otolaryngology, Vol 3, 9th ed. Philadelphia: WB Saunders, 1980; 2045-2050.

41. Courtiss EH, Goldwyn RM, O'Brien JJ. Resection of obstructing inferior nasal turbinates. Plast Reconstr Surg 1978; 62: 249-257.

42. Goodman WS, DeSouza FM (1973) Atrophic rhinitis. Otolaryngol Clin North Am 1973; 6: 773-782.

43. Martinez SA, Nissen AJ, Stock CR, Tesmer T. Nasal turbinate resection for relief of nasal obstruction. Laryngoscope 1983; 93: 871- 875 .

44. Carrie S, Wright RG, Jones AS, Stevens JC, Parker AJ, Yardley MPJ. Long term results of trimming of the inferior turbinates. Clin Otolaryngol 1996; 21: 139 -141.

45. Moore GF, Freeman TJ, Ogren FP, Yonkers AJ. Extended followup of total inferior turbinate resection for relief of chronic nasal obstruction. Laryngoscope 1985; 95: 1095-1099.

46. Moore EJ, Kern EB. Atrophic rhinitis: a review of 242 cases. Am J Rhinol 2001; 15: 355-361.

47. Wight RG, Jones AS, Clegg RT. A comparison of anterior and radical trimming of the inferior turbinates and their effect on nasal resistance to airflow. Clin Otol 1988; 13: 223-226.

48. Fanous N. Anterior turbinectomy. A new surgical approach to turbinate hypertrophy: a review of 220 cases. Arch Otolaryngol Head Neck Surg 1986; 112: 850-852.

49. Mabry RL. "How I do it" - plastic surgery. Practical suggestions on facial plastic surgery. Inferior turbinoplasty. Laryngoscope 1982; 92: 459-461.

50. Mabry RL. Inferior turbinoplasty: Patient selection, technique, and long term consequences. Otolaryngol Head Neck Surg 1988; 98: 60-66.

51. Garth RJ, Cox HJ, Thomas MR. Haemorrhage as a complication of inferior turbinectomy: a comparison of anterior and radical trimming. Clin Otolaryngol 1995; 20: 236-238.

52. Goode RL. Surgery of the turbinates. J Otolaryngol 1978; 7: 262268.

53. Ozenberger JM. Cryosurgery in chronic rhinitis. Laryngoscope 1970; 80: 723-734.

54. Hartley C, Willatt DJ. Cryotherapy in the treatment of nasal obstruction: indications in adults. J Laryngol Otol 1995; 109: 729732.

55. Principato JJ. Chronic vasomotor rhinitis: cryogenic and other surgical modes of treatment. Laryngoscope 1979; 89: 619-638.

56. Bumsted RM. Cryotherapy for chronic vasomotor rhinitis: technique and patient selection for improved results. Laryngoscope 1984; 94: 539-544.

57. Moore JRM, Bicknell PG. A comparison of cryotherapy and submucous diathermy in vasomotor rhinitis. J Laryngol Otol 1980; 94: 1411-1413.

58. Janda P, Sroka R, Baumgartner R, Grevers G, Leunig A. Laser treatment of hyperplastic inferior nasal turbinates. Lasers Surg Med 2001; 28: 404-413.

59. Cook JA, McCombe AW, Jones AS. Laser treatment of rhinitis 1 year follow-up. Clin Otolaryngol 1993; 18: 209-211.

60. Elwany S, Harrison R. Inferior turbinectomy: comparison of four techniques. J Laryngol Otol 1990; 104: 206-209.

61. Pang YT, Willatt DJ. Laser reduction of inferior turbinates in children. Singapore Med J 1995; 36: 514-516.

62. Orabi AA, Sen A, Timms MS, Morar P. Patient satisfaction survey of outpatient-based topical local anaesthetic KTP laser inferior turbinectomy: a prospective study. Am J Rhinol 2007; 21: 198-202. 
63. Wang HK, Tsai YH, Wu YY, Wang PC. Endoscopic potassiumtitanyl-phosphate laser treatment for the reduction of hypertrophic inferior nasal turbinate. Photomed Laser Surg. 204; 22: 173-176.

64. Ferri E, Armato E, Cavaleri S, Capuzzo P, Ianniello F. Argon plasma surgery for treatment of inferior turbinate hypertrophy: a long term follow-up in 157 patients. ORL J Otorhinolaryngol Relat Spec 2003; 65: 206-210.

65. Supiyaphun P, Aramwatanapong P, Kerekhanjanarong V, Sastarasadhit V. KTP laer inferior turbinoplasty: an alternative procedure to treat the nasal obstruction. Auris Nasus Larynx 2003; 30: 59-64.

66. Li KK, Powell NB, Riley RW, Troell RJ, Guilleminault C. Radiofrequency volumetric tissue reduction for treatment of turbinate hypertrophy: a pilot study. Otolaryngol Head Neck Surg 1998; 119: 569-573.

67. Nease CJ, Krempl GA. Radiofrequency treatment of turbinate hypertrophy: a randomized, blinded, placebo-controlled clinical trial. Otolaryngol Head Neck Surg 2004; 130: 291-299.

68. Harsten G. How we do it: radiofrequency-turbinectomy for nasal obstruction symptoms. Clin Otolaryngol 2005; 30: 64-66.

69. Kizilkaya Z, Ceylan K, Emir H, Yavanoglu A, Unlu I, Samim E Akagun MC. Comparison of radiofrequency tissue volume reduction and submucosal resection with microdebrider in inferior turbinate hypertrophy. Otolaryngol Head Neck Surg 2008; 138: 176-181.

70. Cavaliere M, Mottola G, Iemma M. Monopolar and bipolar radiofrequency thermal ablation of inferior turbinates: 20-month follow up. Otolaryngol Head Neck Surg 2007; 137: 256-263.

71. Porter MW, Hales NW, Nease CJ, Krempl GA. Long-term results of inferior turbinate hypertrophy with radiofrequency treatment: a new standard of care? Laryngoscope 2006; 116: 554-557.

72. Barbieri M, Salami A, Mora F, Barbieri A, Cordone MP, Barcalla F, Passali FM, Ralli G, Melloni F, Cassano M, Mora R. High frequency surgery in the treatment of turbinate hypertrophy: 11 years experience. Acta Otorhinolaryngol Ital 2003; 23: 436-439.

73. Rhee CS, Kim DY, Won TB, et al. Changes of nasal function after temperature-controlled radiofrequency tissue volume reduction for the turbinate. Laryngoscope 2001; 111: 153-158.

74. Wu CC, Lee SY, Hsu CJ, Yeh TH. Patients with positive allergen test have less favourable outcome after endoscopic microdebrider assisted inferior turbinoplasty. Am J Rhinol 2008; 22: 20-23.

75. Yanez C. New technique for turbinate reduction in chronic hypertrophic rhinitis, intraturbinate stroma removal using the microdebrider. Oper Tech Otolaryngol Head Neck Surg 1998; 9: 135-137.

76. Mori S, Fujieda S, Yamada T, Kimura Y, Takahashi N, Saito H. Long-term effect of submucous turbinectomy in patients with perennial allergic rhinitis. Laryngoscope 2002; 112: 865-869.

77. Yanez C, Mora N. Inferior turbinate debriding technique: ten-year results. Otolaryngol Head Neck Surg 2008; 138: 170-175.
78. Ikeda K, Oshima T, Suzuki M, Suzuki H, Shimomura A. Functional inferior turbinosurgery (FITS) for the treatment of resistant chronic rhinitis. Acta Otolaryngol 2006; 126: 739-745.

79. Ikeda K, Yokio H, Saito T, Kawano K, Yao T, Furukawa M. Effect of resection of the posterior nasal nerve on functional and morphological changes in the inferior turbinate mucosa. Acta Otolaryngologica 2008; 128: 1337-1341.

80. Wengraf CL, Gleeson MJ, Siodlak MZ. The stuffy nose: a comparative study of two common methods of treatment. Clin Otolaryngol 1986; 11: 61-68.

81. Rakover Y, Rosen G. A comparison of partial inferior turbinectomy and cryosurgery for hypertrophic inferior turbinates. J Laryngol Otol 1996; 110: 732-735.

82. Cole Ph, Chaban R, Naito K. The obstructive nasal septum. Archives of Otolaryngology, Head Neck Surgery 1988; 114: 410412.

83. Hilberg O, Cryner LF, Pedersin OF. Turbinate hypertrophy evaluation of the nasal cavity by acoustic rhinomanometry. Arch Otolaryngol Head Neck Surg 1990; 117: 284-289.

84. Illum P. Septoplasty and compensatory inferior turbinate hypertrophy: long-term results after randomised turbinoplasty. Eur Arch Otorhinolaryngol 1997; 254(suppl 1): S89-S92.

85. White A, Murray JAM. Intranasal adhesion formation following surgery for chronic nasal obstruction. Clin Otolaryngol 1988; 13: 139-143.

86. Nunez DA, Bradley PJ. A randomised clinical trial of turbinectomy for compensatory turbinate hypertrophy in patients with anterior septal deviations. Clin Otolaryngol 2000; 25: 495-498.

87. Kim DH, Park HY, Kim HS et al. Effect of septoplasty on inferior turbinate hypertrophy. Arch Otolaryngol Head Neck Surg 2008; 134: 419-423.

88. Stewart MG, Smith TL, Weaver EM et al. Outcomes after nasal septoplasty: results from the Nasal Obstruction Septoplasty Effectiveness (NOSE) study. Otolaryngol Head Neck Surg 2004; 130: $283-290$

Mr D. J. Willatt

ENT Department

Salford Royal NHS Foundation Trust

University Hospital

Eccles Old Road

Salford M6 8HD

United Kingdom

Tel: +44-161-206 4758

Fax +44-161-206 4723

Email d.j.Willatt@talk21.com 